\title{
Trends of Antimicrobial Resistance in Typhoidal Strains of Salmonella in a Tertiary Care Hospital in Pakistan
}

\author{
Aqsa Aslam ${ }^{1}$, Sahibzada Ahmed Kharal ${ }^{2}$, Maria Aslam ${ }^{3}$, Almas Raza 4 \\ 1. Microbiology, National University of Medical Sciences, Rawalpindi, PAK 2. Family Medicine, Forrest Family Practice, \\ Bunbury, AUS 3. Hematology, Sharif Medical and Dental College, Lahore, PAK 4. Microbiology, Pak Red Crescent \\ Medical and Dental College, Lahore, PAK
}

Corresponding author: Aqsa Aslam, aksaakhawar@gmail.com

\begin{abstract}
Introduction: Enteric fever or typhoid fever is a major public health issue affecting greater than 27 million individuals globally and is responsible for greater than 200,000 deaths per year. Due to the extensive overuse of antimicrobials, the world is moving toward a pre-antibiotic era. The emergence and transmission of antibiotic-resistant Salmonella species are a global threat and a serious concern in developing countries such as Pakistan. This study aimed to determine the trends in antimicrobial resistance (AMR) of typhoidal strains of Salmonella in a tertiary care hospital in Pakistan.
\end{abstract}

Materials and Methods: It was a descriptive, cross-sectional study conducted in the pathology department of Sharif City Hospital, Lahore, after approval by the ethical committee of the institution. A total of 50 blood culture specimens positive for Salmonella typhi and Salmonella paratyphi from January 2019 to March 2020 were included by the non-probability consecutive sampling technique. The samples were processed by conventional bacteriological methods for isolation and identification. The antimicrobial susceptibility testing was done by the Kirby-Bauer disc diffusion method as recommended by the Clinical and Laboratory Standard Institute (CLSI). The statistical package for social sciences (SPSS, IBM Corp., Armonk, NY) version 25 was used for data entry and analysis.

Results: Among the first-line drugs (ampicillin, chloramphenicol, and trimethoprim-sulfamethoxazole), 70\% of strains were resistant, and only $30 \%$ strains were sensitive to them. Among the cephalosporins, $52 \%$ strains were sensitive to ceftriaxone, and $48 \%$ strains were sensitive to ceftazidime, cefotaxime, and cefepime. Twenty-four percent of strains were sensitive to ciprofloxacin. Only $50 \%$ of strains were sensitive to ampicillin-sulbactam, and $92 \%$ of strains were sensitive to piperacillin-tazobactam. All the strains were $100 \%$ sensitive to imipenem and meropenem; $96 \%$ of strains were sensitive to co-amoxiclav, doxycycline, and azithromycin. The frequency of multidrug-resistant (MDR) and extensively drug-resistant (XDR) Salmonella species was $16 \%$ and $54 \%$.

Review began $10 / 27 / 2020$ Review ended 12/13/2020 Published 01/12/2021

\section{() Copyright 2021}

Aslam et al. This is an open access article distributed under the terms of the Creative Commons Attribution License CC-BY 4.0., which permits unrestricted use, distribution, and reproduction in any medium, provided the original author and source are credited.
Conclusion: The increasing frequency of MDR and XDR Salmonella species in Pakistan is a major concern. A significant percentage of the typhoidal strains of Salmonella is resistant to the first-line (16\%) and secondline (54\%) antibiotics. Carbapenems and azithromycin are the last resort of therapy in such cases.

Categories: Pathology, Infectious Disease

Keywords: typhoidal strains of salmonella, antimicrobial resistance, salmonella typhi, salmonella paratyphi

\section{Introduction}

Enteric fever or typhoid fever is an acute, fatal febrile illness with a mortality rate of $1 \%$ to $3 \%$. It is a major public health issue affecting greater than 27 million individuals globally and is responsible for greater than 200,000 deaths per year $[1,2]$. It causes significant morbidity and mortality globally, especially in developing countries. Its causative organisms are Salmonella typhi and Salmonella paratyphi $A, B$, and $C$ [3]. The transmission is through the fecal-oral route mainly through contaminated water. Patients with enteric fever present with high-grade fever, malaise, headache, anorexia, nausea, abdominal pain, and diarrhea or constipation. Physical examination reveals hepatomegaly, splenomegaly, and relative bradycardia. The complications of this infection are intestinal perforation, bleeding, peritonitis, and meningoencephalitis. The diagnostic test for enteric fever is blood culture or bone marrow culture in the first week [1-4]. The mainstay of treatment is antibiotics. The first-line antibiotics are ampicillin, chloramphenicol, and trimethoprim-sulfamethoxazole. The second-line antibiotics are fluoroquinolones. Ceftriaxone, azithromycin, and carbapenems are used when isolates show resistance to first- and second-line antibiotics. The effective ways for the prevention of enteric fever are vaccination, hand hygiene, improved sanitation, and the use of clean water [5].

The genus Salmonella is a facultative anaerobe, gram-negative bacillus, and is classified into two 
species, Salmonella enterica and Salmonella bongori [1]. It is a member of the Enterobacteriaceae family [2]. Salmonella enterica subspecies enterica has 2,600 serovars, out of which four serovars are clinically important. Salmonella typhi and Salmonella paratyphi cause enteric fever, while Salmonella typhimurium and Salmonella enteritidis cause non-typhoidal salmonellosis [1,2].

Salmonellosis is common in the developing world including Asia, Africa, and South America [6]. According to a study, enteric fever has a prevalence of 29.3 per 100,000 person-years in China, 24.2 in Vietnam, 180.3 in Indonesia, 493.5 in India, and 412.9 in Pakistan [3]. Multidrug-resistant Salmonella has been reported from South Asia. Multidrug-resistant Salmonella is strain-resistant to first-line antibiotics (ampicillin, chloramphenicol, and trimethoprim-sulfamethoxazole) [6]. Nowadays, extensively drug-resistant (XDR) Salmonella has emerged as an important health issue in Pakistan. These isolates can spread globally via conjugation or transposons and transform MDR strains to XDR strains. Salmonella strains are labeled as XDR when they show resistance to first-line antibiotics, fluoroquinolones, and third-generation cephalosporins [7]. Extensively drug-resistant Salmonella was first reported in 2016 in Hyderabad, Sindh [8]. The analysis of the genome sequence of these strains showed that they had an H58 haplotype [6]. The treatment of choice for these strains is azithromycin and meropenem. Azithromycin resistance has also been documented in India [9]. The antibiotic resistance genes (AMR) in the H58 haplotype are associated with an IncHI1 plasmid. These genes include blaTEM-1 conferring resistance to ampicillin, dfrA7, sul1, sul2mediating resistance to trimethoprim-sulfamethoxazole, and catA1 causing chloramphenicol resistance. Ceftriaxone resistance is conferred by the extended-spectrum $\beta$ lactamase (ESBL) gene. Resistance to fluoroquinolones is caused by mutations in the DNA gyrase (gyrA and gyrB) and topoisomerase IV genes [10].

The World Health Organization (WHO) reported 5274 cases of XDR Salmonella typhi cases in Sindh from 2016 to 2018. Seventy-six percent of these cases were from Karachi city followed by Hyderabad (27\%) and other Sindh districts $(4 \%)[6,11]$. Instead of the awareness campaigns and infection control measures implemented by the government, the frequency of XDR Salmonella typhi cases raised from 2017 to 2018 [7]. Five cases of XDR Salmonella typhi were reported from the United States, one case from the United Kingdom, and one case from Canada. But all of them traveled to Pakistan [12,13]. The study aimed to determine the trends in antimicrobial resistance (AMR) of typhoidal strains of Salmonella in a tertiary care hospital in Pakistan. Due to the extensive overuse of antimicrobials, the world is moving toward a pre-antibiotic era. The emergence and transmission of antibiotic-resistant Salmonella species are a global threat and a serious concern in developing countries such as Pakistan. Our study will add data and set a priority for future research in this aspect. This study will help in the scaling-up of policies at the government level in Pakistan and other countries with a high prevalence of disease to combat the rising burden of enteric fever. In addition, antimicrobial surveillance is poor, and antibiotic stewardship policies should be implemented.

\section{Materials And Methods}

It was a descriptive, cross-sectional study done in the Pathology Department of Sharif City Hospital, Lahore, after approval by the ethical committee of the institution. A total of 1510 blood culture specimens positive for Salmonella typhi and Salmonella paratyphi from January 2019 to March 2020 were included by the nonprobability consecutive sampling technique.

The samples for blood culture were received in the microbiology section of the laboratory in blood culture bottles containing tryptic soya broth and polyanethol sulfonate. The bottles were placed in the incubator at $35^{\circ} \mathrm{C}$ for five days. The first subculture was performed on blood agar and MacConkey agar after 48 hours. After the first culture was negative, the second subculture was inoculated on blood agar and MacConkey agar in the following 72 hours. Salmonella species produce non-lactose fermenting colonies on MacConkey agar and were identified by colony morphology, gram staining, and oxidase test. In the case of gramnegative rods and negative oxidase test, they were further identified at the species level using the analytical profile index (API) and slide agglutination test with antisera. Direct gram staining from blood culture bottles and reculture of specimens were also done to confirm the presence of the causative organism. The antibiotic susceptibility testing of the causative organisms was performed using the Kirby-Bauer disc diffusion method. The suspension of the causative organism was made by mixing three to four colonies of the organism in the normal saline and was matched with the $0.5 \mathrm{McFarland}$ turbidity standard. The suspension was evenly applied on the Mueller-Hinton agar plates using the sterile swab, and antibiotic disks were placed on the plates with an aseptic technique. The plates were incubated for $18-24$ hours at $35^{\circ} \mathrm{C}$, and the zone diameters of antibiotics were evaluated according to the Clinical and Laboratory Standards Institute (CLSI) 2019 [14]. The antibiotics were reported into these categories: susceptible, resistant, or intermediate. The antibiotic panel applied for Salmonella included ampicillin (AMP), trimethoprim-sulfamethoxazole (SXT), chloramphenicol (C), ciprofloxacin (CIP), ceftazidime (CAZ), cefotaxime (CTX), ceftriaxone (CRO), cefepime (FEP), imipenem (IPM), meropenem (MEM), piperacillin-tazobactam (TZP), ampicillin-sulbactam (SAM), co-amoxiclav (AMC), doxycycline (DO), and azithromycin (AZM).

The Statistical Package for the Social Sciences (SPSS, IBM Corp., Armonk, New York) software version 25.0 was used for data analysis. The age of the patients was expressed as mean and standard deviation. The gender, respective department of patients, the prevalence of positive blood cultures, and antibiotic susceptibility pattern of the isolates were expressed as frequency and percentages. 


\section{Cureus}

\section{Results}

A total of 1510 blood specimens were received in the pathology laboratory, out of which 50 (3.31\%) blood cultures were positive for Salmonella. Out of the 50 positive cultures, 47 (94\%) were Salmonella typhi, and three (6\%) were Salmonella paratyphi. Among the patients with positive cultures, 34 (68\%) were males, while $16(32 \%)$ were females. The majority (60\%) of the patients with positive cultures were children ranging from one to 10 years old followed by 11 to 20 years (26\%) and 21 to 30 years of age group (8\%). Four percent of patients with positive cultures were below one year old and $2 \%$ were above 30 years. The distribution of the positive cultures received from different departments of the hospital includes $26(52 \%)$ from the pediatrics department, 11 (22\%) from the various outpatient departments, eight (16\%) from the medicine department, and five (10\%) from the emergency department. Thirty-one (62\%) positive cases were reported from June 2019 to September 2019, nine (18\%) cases from March to May 2019, and five (10\%) cases from October 2019 to February 2020. The frequency of Salmonella-positive cultures during the study duration is shown in Figure 1.

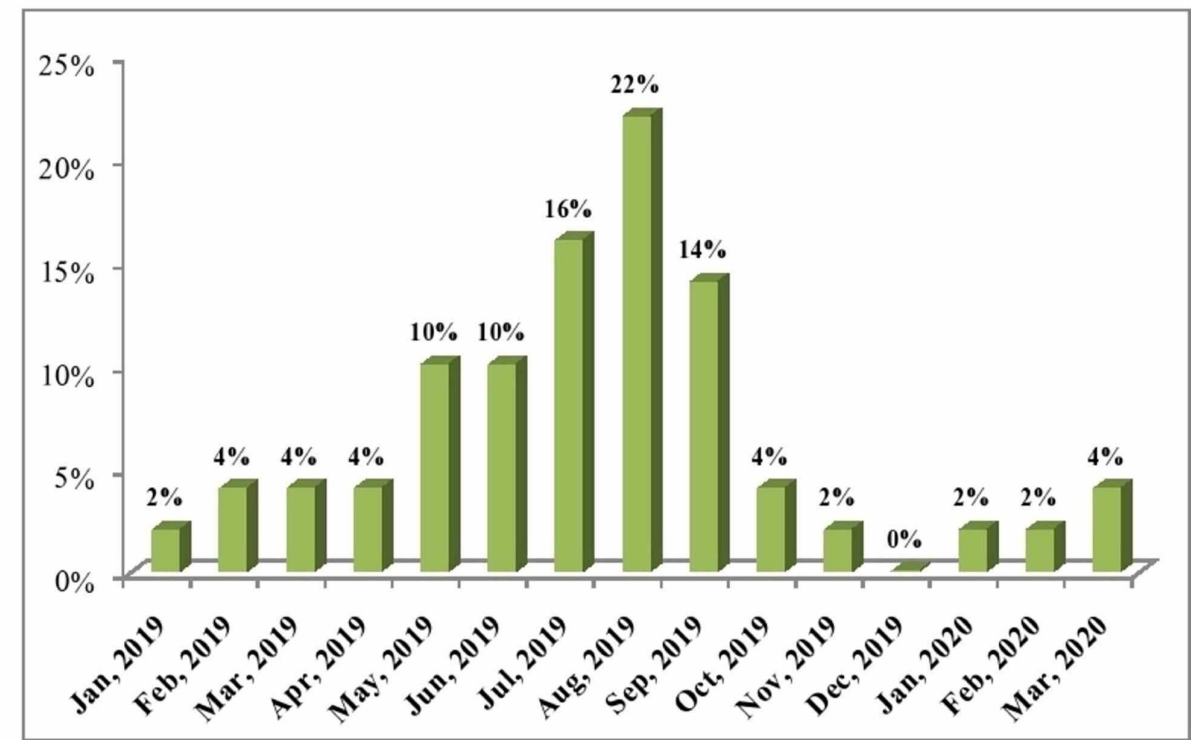

FIGURE 1: Frequency of Salmonella-Positive Cultures in the 15-Month Period

Among the first-line drugs (ampicillin, chloramphenicol, and trimethoprim-sulfamethoxazole), $70 \%$ of strains were resistant, and only $30 \%$ strains were sensitive to them. Among the cephalosporins, $52 \%$ strains were sensitive to ceftriaxone, and $48 \%$ strains were sensitive to ceftazidime, cefotaxime, and cefepime. Ciprofloxacin resistance was noted in $76 \%$ of strains. Only $50 \%$ of strains were sensitive to ampicillinsulbactam, and $92 \%$ of strains were sensitive to piperacillin-tazobactam. All the strains were $100 \%$ sensitive to imipenem and meropenem; $96 \%$ of strains were sensitive to co-amoxiclav, doxycycline, and azithromycin. The frequency of MDR Salmonella was 16\%, and XDR Salmonella was 54\%. Figure 2 shows the antimicrobial susceptibility pattern of Salmonella typhi and Salmonella paratyphi. 


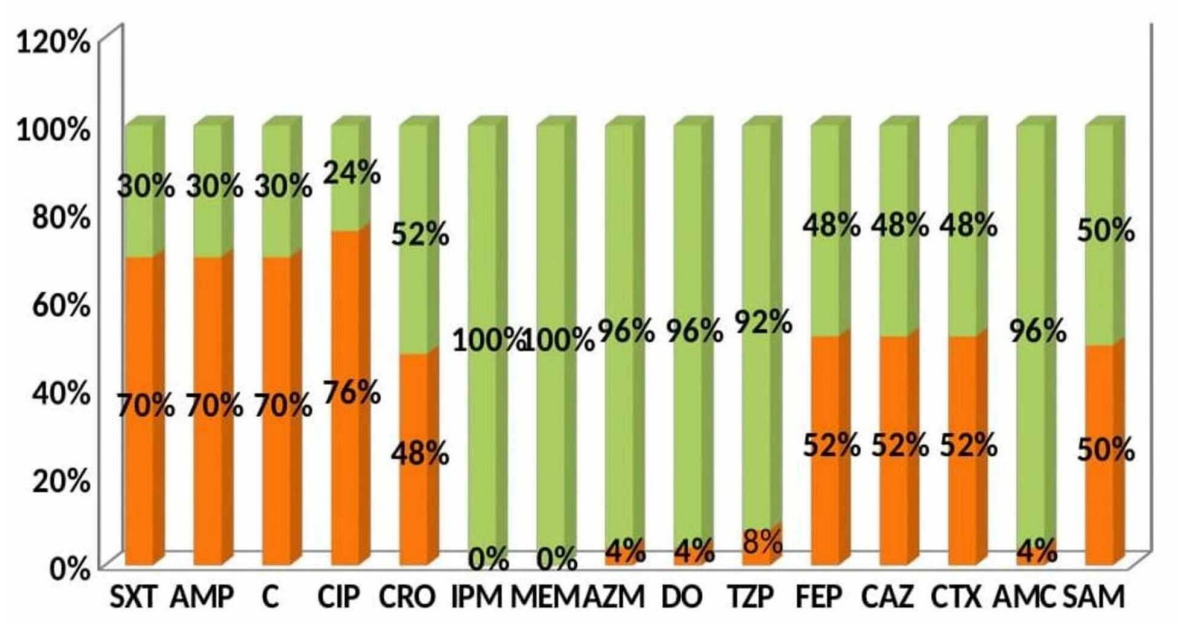

nesistant $n$ Sensitive

FIGURE 2: Antimicrobial Susceptibility Pattern of Salmonella Typhi and Salmonella Paratyphi

SXT, Trimethoprim-sulfamethoxazole; AMP, ampicillin; C, chloramphenicol; CIP, ciprofloxacin; CRO, ceftriaxone; IPM, imipenem; MEM, meropenem; AZM, azithromycin; DO, doxycycline; TZP, piperacillintazobactam; FEP, cefepime; CAZ, ceftazidime; CTX, cefotaxime; AMC, co-amoxiclav; SAM, ampicillinsulbactam

\section{Discussion}

Enteric fever is a common disease in developing countries due to several factors such as inadequate healthcare systems and poor sanitation [15]. Antibiotic resistance has become an important concern in treating the disease. Salmonella strains resistant to first-line antibiotics and third-generation cephalosporins have been isolated in Asia. The most common MDR haplotype of Salmonella typhi is H58, and it is spreading worldwide [10].

In our study, $94 \%$ of the Salmonella strains were Salmonella typhi, and only $6 \%$ were Salmonella paratyphi A. In another study, Salmonella typhi accounted for $84.5 \%$, and Salmonella paratyphi $A$ for $15.1 \%$ of the Salmonella strains. Salmonella paratyphi $B$ and $C$ were reported from only one case each, respectively [16]. In our study, most (68\%) of patients with positive cultures were males. Similarly, Iyer et al. reported that $53.2 \%$ of the typhoidal cases were in males [16]. The majority (60\%) of the patients with positive cultures were children with their ages ranging from 1 to 10 years followed by 11 to 20 years (26\%) in our study. A study showed $61.9 \%$ of cases of Salmonella in five to 15 years of age and $23.4 \%$ cases in two to five years of age [16]. In our study, $62 \%$ of positive cases were reported from June to September $2019,18 \%$ cases from March 2019 to May 2019, and 10\% cases from October 2019 to February 2020. Similarly, most of the cases (36.4\%) were reported from June to September in a study [16].

In our study, $70 \%$ of strains were resistant to ampicillin, chloramphenicol, and trimethoprimsulfamethoxazole. Among the cephalosporins, $52 \%$ strains were sensitive to ceftriaxone, and $48 \%$ strains were sensitive to ceftazidime, cefotaxime, and cefepime. Ciprofloxacin resistance was noted in $76 \%$ of strains. Only $50 \%$ of strains were sensitive to ampicillin-sulbactam, and $92 \%$ of strains were sensitive to piperacillin-tazobactam. All the strains were $100 \%$ sensitive to imipenem and meropenem; $96 \%$ of strains were sensitive to co-amoxiclav, doxycycline, and azithromycin. The frequency of MDR Salmonella was $16 \%$, and XDR Salmonella was $54 \%$.

In a report by Chatham-Stephens et al. in the United States, Salmonella species causing enteric fever were isolated from 3538 blood cultures. Out of these, $65 \%$ were resistant to fluoroquinolones and $12 \%$ were MDR; 244 culture-positive patients had traveled to Pakistan, out of which $79 \%$ were fluoroquinolone-resistant and $50 \%$ were MDR [12]. In a study by Iyer et al., $100 \%$ of strains were sensitive to ceftriaxone and azithromycin [16]. In another study done in Shifa International Hospital, Islamabad, the frequency of MDR and XDR strains was $14 \%$ and $4.01 \%$, respectively. A study conducted in India showed that all the typhoidal strains of Salmonella were sensitive to ceftriaxone and azithromycin. Ninety-four strains were also sensitive to chloramphenicol, and only $3.6 \%$ were sensitive to ofloxacin [17].

Laghari et al. reported $81.6 \%$ and 100\% ampicillin-sensitive Salmonella typhi and Salmonella paratyphi, 
respectively, from Jamshoro, Southern Pakistan. Cotrimoxazole was sensitive in $96.4 \%$ Salmonella typhi and $100 \%$ Salmonella paratyphi A. Among the cephalosporins, $65.8 \%$ of the Salmonella typhi and $96.1 \%$ of Salmonella paratyphi A were ceftriaxone-sensitive. Ninety percent of Salmonella typhi and $94.2 \%$ of Salmonella paratyphi A were sensitive to cefotaxime. Ciprofloxacin was sensitive in $50.1 \%$ Salmonella typhi and 67.3\% Salmonella paratyphi A. Imipenem showed sensitive zones in 87.8\% Salmonella typhi and 96.1\% Salmonella paratyphi A. Azithromycin was sensitive in $94.6 \%$ and $100 \%$ Salmonella typhi and Salmonella paratyphi A, respectively. The prevalence of MDR and XDR Salmonella typhi and Salmonella paratyphi was $2.6 \%$ and $0.9 \%$ [18]. Another study in Pakistan reported that the frequency of MDR Salmonella typhi was $76 \%$ and Salmonella paratyphi was 34\%. The frequency of XDR Salmonella typhi was $48 \%$ [19].

In a study conducted in India, ampicillin resistance was noted in $29.47 \%$ Salmonella typhi, co-trimoxazole resistance in $17.89 \%$ strains, and chloramphenicol resistance in 28.42 strains. All the strains were sensitive to imipenem [15]. Another study done in India reported that all the Salmonella typhi strains were sensitive to imipenem, and $97.67 \%$ of strains were sensitive to meropenem. Eighty-three percent of Salmonella typhi were sensitive to ampicillin-sulbactam, $91 \%$ sensitive to ceftazidime, $90.6 \%$ sensitive to ceftriaxone, $57.5 \%$ sensitive to ciprofloxacin, and 57.5\% sensitive to cotrimoxazole. The MDR Salmonella typhi were present in $44 \%$ of the cases [20].

The management of drug-resistant typhoidal strains of Salmonella has become a great challenge in Pakistan. The policies should be implemented at the government level to combat the rising burden of enteric fever. In addition, antimicrobial surveillance is poor, and antibiotic stewardship policies should be scaled-up. The use of antibiotics should be kept to a minimum. Antibiotics should not be available over the counter without the prescription of the doctor. Carbapenems and azithromycin should not be prescribed routinely unless the strain is resistant to first-line drugs, ciprofloxacin, and ceftriaxone on the antibiotic susceptibility report. However, the study has some limitations. A multicenter study should be conducted with a larger sample size. The antibiotic sensitivity testing should be done using the minimum inhibitory concentration (MIC).

\section{Conclusions}

The increasing frequency of MDR and XDR Salmonella species in Pakistan is a major concern. A significant percentage of the typhoidal strains of Salmonella is resistant to first-line (16\%) and second-line (54\%) antibiotics. Carbapenems and azithromycin are the last resort of therapy in such cases.

\section{Appendices}

RESEARCH PARTICIPANT CONSENT FORM

\section{FOR RESEARCH PROJECT}

Serial No:

Date:

Study Center: Sharif Medical and Dental College, Lahore

PART I: Information Sheet

Introduction: The participants will be explained in a language that they can understand easily. After a brief introduction, the participants are explained that they are invited to participate in the research. They will be informed that they may talk to anyone they feel comfortable with about the research and that they can take time to reflect on whether they want to participate or not. Assure the participant that if they do not understand some of the words or concepts that you will take time to explain to them as you go along and that they can ask questions now or later.

Purpose: The study aimed to determine the trends in antimicrobial resistance (AMR) of typhoidal strains of Salmonella in a tertiary care hospital in Pakistan. Due to the extensive overuse of antimicrobials, the world is moving toward a pre-antibiotic era. The emergence and transmission of antibiotic-resistant Salmonella species are a global threat and serious concern in developing countries such as Pakistan. This study will help in the scaling-up of policies at the government level in Pakistan and other countries with a high prevalence of disease to combat the rising burden of enteric fever.

Procedure: This study will require $10 \mathrm{ml}$ blood sample from each patient through venipuncture using aseptic measures. All the required particulars of the patient will be recorded on the proforma sheet. Each sample will be processed by culture, gram staining, antibiotic susceptibility testing, and biochemical tests in the laboratory.

Voluntary Participation: Your participation in the study is entirely voluntary. Whether you choose to participate or not, all the services you receive at this hospital will continue in the same manner.

Time: Three to five minutes will be required for every patient to participate in this study. 
Possible benefits: To diagnose enteric fever and determine the antibiotic susceptibility pattern of Salmonella.

Possible risks or discomfort: Venipuncture is a reasonably safe medical technique. However, bruising, hematoma formation and vasovagal syncope can occur in a few persons. The healthcare provider will try his/her best to decrease the chances of these complications but if any one of these occurs unexpectedly, you will be provided with immediate adequate medical care.

Financial Consideration: There will be no financial compensation for participation in the study. The financial benefit gained is free of cost testing for the participant.

Confidentiality: Your identity will not be disclosed, and all the records will be kept confidential. The result of the study including laboratory or any other data may be published.

Sharing the results: The results of your tests will be shared with you.

Withdrawal from Participation: You will have the right to refuse to participate in the study even if the consent form is signed. It will not affect your treatment or rights as a patient here.

Who to Contact: Provide the name and contact information of someone who is involved, informed, and accessible (a local person who can actually be contacted).

This proposal has been reviewed and approved by the institutional review board (IRB), which is a committee whose task is to make sure that research participants are protected from harm.

PART II: Certificate of Consent

I have read the foregoing information, or it has been read to me. I have had the opportunity to ask questions about it, and any questions that I have asked have been answered to my satisfaction. I consent voluntarily to participate as a participant in this research.

Name of Participant: Age:

Signature of Participant:

If illiterate

If the research participant is illiterate, a literate witness will sign (witness is selected by the participant and has no connection to the research team). Participants who are illiterate should include their thumbprint as well.

I have witnessed the accurate reading of the consent form to the potential participant, and the individual has had the opportunity to ask questions. I confirm that the individual has given consent freely.

Name of Witness Participant:

Signature of Witness:

Thumbprint of Participant:

\section{Additional Information}

\section{Disclosures}

Human subjects: Consent was obtained or waived by all participants in this study. Sharif Medical and Dental College, Lahore Ethical Committee issued approval SMDC/SMRC/68-18. The institutional Ethical Committee of Sharif Medical and Dental College (SMDC), Lahore has reviewed and approved the study to be conducted in the Pathology department of SMDC (Study approval number: SMDC/SMRC/68-18). Animal subjects: All authors have confirmed that this study did not involve animal subjects or tissue. Conflicts of interest: In compliance with the ICMJE uniform disclosure form, all authors declare the following: Payment/services info: All authors have declared that no financial support was received from any organization for the submitted work. Financial relationships: All authors have declared that they have no financial relationships at present or within the previous three years with any organizations that might have an interest in the submitted work. Other relationships: All authors have declared that there are no other relationships or activities that could appear to have influenced the submitted work. 


\section{References}

1. Saleh S, Van Puyvelde S, Staes A, et al.: Salmonella typhi, paratyphi A, enteritidis and typhimurium core proteomes reveal differentially expressed proteins linked to the cell surface and pathogenicity. PLoS Negl Trop Dis. 2019, 13:0007416. 10.1371/journal.pntd.0007416

2. Zhang XL, Jeza VT, Pan Q: Salmonella typhi: from a human pathogen to a vaccine vector . Cell Mol Immunol. 2008,5, 91:7. 10.1038/cmi.2008.11

3. Das JK, Hasan R, Zafar A, et al.: Trends, associations and antimicrobial resistance of Salmonella typhi and paratyphi in Pakistan. Am J Trop Med Hyg. 2018 Sep, 99:48-54. 10.4269/ajtmh.18-0145

4. Gotuzzo E: Typhoid fever: a current problem. Int J Infect Dis. 2018, 73:46-7. 10.1016/j.ijid.2018.04.3528

5. Crump JA, Sjolund-Karlsson M, Gordon MA, Parry CM: Epidemiology, clinical presentation, laboratory diagnosis, antimicrobial resistance and antimicrobial management of invasive Salmonella infections. Clin Microbiol Rev. 2015, 28:901-37. 10.1128/CMR.00002-15

6. Saeed N, Usman M, Khan EA: An overview of extensively drug-resistant Salmonella typhi from a tertiary care hospital in Pakistan. Cureus. 2019, 11:5663. 10.7759/cureus.5663

7. Typhoid fever - Islamic Republic of Pakistan . (2018). Accessed: April 1, 2019: https://www.who.int/csr/don/27-december-2018-typhoid-pakistan/en/.

8. Yousafzai MT, Qamar FN, Shakoor S, et al.: Ceftriaxone-resistant Salmonella typhi outbreak in Hyderabad city of Sindh, Pakistan: high time for the introduction of typhoid conjugate vaccine. Clin Infect Dis. 2019, 68:16-21. 10.1093/cid/ciy877

9. Manesh A, Balaji V, Kumar DRN, Rupali P: A case of clinical and microbiological failure of azithromycin therapy in Salmonella enterica serotype typhi despite low azithromycin MIC. Int J Infect Dis. 2017, 54:62-3. 10.1016/j.ijid.2016.11.409

10. Klemm EJ, Shakoor S, Page AJ, et al.: Emergence of an extensively drug-resistant Salmonella enterica serovar typhi clone harboring a promiscuous plasmid encoding resistance to fluoroquinolones and thirdgeneration cephalosporins. mBio. 2018, 9:00105-18. 10.1128/mBio.00105-18

11. Akram J, Khan AS, Khan HA, et al.: Extensively drug-resistant typhoid: evolution, prevention and its management. Biomed Res Int. 2020, 2020:1-7. 10.1155/2020/6432580

12. Chatham-Stephens K, Medalla F, Hughes M, et al.: Emergence of extensively drug-resistant Salmonella typhi infections among travelers to or from Pakistan - United States, 2016-2018. MMWR Morb Mortal Wkly Rep. 2019, 68:11-3. 10.15585/mmwr.mm6801a3

13. Wong W, Rawahi HA, Patel S, et al.: The first Canadian pediatric case of extensively drug-resistant Salmonella typhi originating from an outbreak in Pakistan and its implication for empiric antimicrobial choices. IDCases. 2018, 15:00492. 10.1016/j.idcr.2019.e00492

14. Clinical and Laboratory Standards Institute. Performance standards for antimicrobial susceptibility testing; twenty-ninth informational supplement. (2019). Accessed: September 15, 2020: http:////www.clsi.org.

15. Nagshetty K, Shivannavar T, Channappa ST, Gaddad SM: Antimicrobial susceptibility of Salmonella typhi in India. J Infect Dev Ctries. 2010, 4:70-3. 10.3855/iidc.109

16. Iyer RN, Jangam RR, Jacinth A, Venkatalakshmi A, Nahdi FB: Prevalence and trends in the antimicrobial susceptibility pattern of Salmonella enterica serovars typhi and paratyphi A among children in a pediatric tertiary care hospital in South India over a period of ten years: a retrospective study. Eur J Clin Microbiol Infect Dis. 2017, 36:2399-2404. 10.1007/s10096-017-3073-X

17. Patil N, Mule P: Sensitivity pattern of Salmonella typhi and paratyphi A isolates to chloramphenicol and other anti-typhoid drugs: an in-vitro study. Infect drug resist. 2019, 12:3217-25. 10.2147/IDR.S204618

18. Laghari GS, Hussain Z, Hussain SZM, Kumar H, Mazharuddin SM, Haq A: Antimicrobial susceptibility patterns of Salmonella species in Southern Pakistan. Cureus. 2019, 11:4379. 10.7759/cureus.4379

19. Hussain A, Satti L, Hanif F, et al.: Typhoidal Salmonella strains in Pakistan: an impending threat of extensively drug-resistant Salmonella typhi. Eur J Clin Microbiol Infect Dis. 2019, 38:2145-9. 10.1007/s10096-019-03658-0

20. Chandane P, Gandhi A, Bowalekar S: Study of antibiotic susceptibility pattern of Salmonella typhi in children suffering from enteric fever. Ann Trop Med Public Health. 2017, 10:440-3. 10.4103/ATMPH.ATMPH_103_17 